\title{
MarA, SoxS and Rob function as virulence factors in an Escherichia coli murine model of ascending pyelonephritis
}

\author{
Paul Casaz, ${ }^{1} \dagger$ Lynne K. Garrity-Ryan, ${ }^{1}$ David McKenney, ${ }^{1}$ \\ Caroline Jackson, ${ }^{1}$ Stuart B. Levy, ${ }^{1,2}$ S. Ken Tanaka ${ }^{1}$ \\ and Michael N. Alekshun ${ }^{1} \ddagger$ \\ ${ }^{1}$ Paratek Pharmaceuticals, Inc., 75 Kneeland Street, Boston, MA 02111, USA \\ ${ }^{2}$ Center for Adaptation Genetics and Drug Resistance, Tufts University School of Medicine, \\ 136 Harrison Avenue, Boston, MA 02111, USA
}

Correspondence

Stuart B. Levy

stuart.levy@tufts.edu

Received 27 July 2006

Revised 13 September 2006

Accepted 15 September 2006

\section{INTRODUCTION}

The AraC family of transcription factors is composed of more than 1000 members (Alekshun \& Levy, 2004), many of which have well-known roles as virulence factors (Finlay \& Falkow, 1997). ExsA from Pseudomonas aeruginosa regulates a type III secretion system (TTSS) (Hauser et al., 1998), Yersinia spp. LcrF (VirF) and YbtA control a TTSS (Flashner et al., 2004) and yersiniabactin (siderophore) (Fetherston et al., 1996) biosynthesis, respectively, and ToxT from Vibrio cholerae governs the synthesis of cholera toxin and toxin coregulated pili (Champion et al., 1997). Inactivation of genes specifying AraC family members [e.g. BfpT, ToxT, LcrF (VirF), Rv1931c, ExsA, Sp1433 and MarA] attenuates virulence in human subjects (Bieber et al., 1998) and a variety of animal infection models (Champion et al., 1997; Flashner et al., 2004; Frota et al., 2004; Hauser et al., 1998; Hava \& Camilli, 2002; Randall \& Woodward, 2001).

Thus, in addition to a primary role in virulence, it is assumed that many members of the AraC family play larger roles in affecting the overall physiology of the bacterial cell.

†Present address: Massachusetts Biologics Laboratories, 305 South Street, Jamaica Plain, MA 02130, USA.

‡Present address: Schering-Plough Research Institute, 2015 Galloping Hill Road, Kenilworth, NJ 07033-0530, USA.

Abbreviations: TTSS, type III secretion system; UPEC, uropathogenic E. coli; UTI, urinary tract infection; VUR, vesicoureteral reflux.
Notably, genomic array experiments have shown that $P$. aeruginosa ExsA and $V$. cholerae ToxT regulate the expression of a large collection of genes termed regulons (Bina et al., 2003; Wolfgang et al., 2003).

Escherichia coli MarA and SoxS were originally identified based on their ability to control multiple antibiotic resistance (Mar) (George \& Levy, 1983a, b), and susceptibility to superoxide and other oxidative stress agents ( Wu \& Weiss, 1991), respectively. Experiments with Rob, a MarA and SoxS paralogue, showed that it could function in a similar manner (Ariza et al., 1995). Subsequent data have documented multidrug-resistant clinical strains of E. coli (Linde et al., 2000; Maneewannakul \& Levy, 1996) and Salmonella enterica serovar Typhimurium (S. typhimurium) (Koutsolioutsou et al., 2001) that constitutively express AraC family members. It is therefore surmised that these proteins may play a role in the infectious process.

Although the soxS and mar loci are expressed by $S$. typhimurium within macrophages (Valdivia \& Falkow, 1996) and J774-A.1 (macrophage-like) cells (Eriksson et al., 2003), initial attempts to demonstrate experimentally an effect on virulence in vivo have been unsuccessful. Sulavik et al. (1997) used a lethal infection model to investigate the virulence of strains lacking soxS. Van der Straaten and colleagues (2004) failed to find differences during in vivo growth of wild-type organisms compared with strains lacking ramA (specifying another AraC protein), soxS or 
both genes. Randall \& Woodward (2001), however, found that S. typhimurium DT104 lacking marA was less likely to colonize the spleens and caeca of infected chicks.

MarA, SoxS and Rob each regulate the expression of multiple genes (called the MarA, SoxS and Rob regulons) in E. coli (Barbosa \& Levy, 2000; Bennik et al., 2000; Pomposiello et al., 2001), S. typhimurium (Pomposiello \& Demple, 2000) and, by inference, other members of the Enterobacteriaceae. This regulation is achieved by the binding of the transcription factor to a degenerate sequence, called the 'marbox' or 'soxbox' (Martin \& Rosner, 2003), which is located within the promoter of the regulated gene. Since MarA has been found recently to act as a transcription repressor (Schneiders et al., 2003), it is more precise to state that the location of the marbox can be found among both promoter and operator sequences. The degeneracy of the marbox/soxbox allows for the differential regulation of individual genes by MarA, SoxS and Rob (Martin et al., 2000; Martin \& Rosner, 2003). Thus, experiments investigating phenotypes attributed to these proteins should be performed with strains lacking single and multiple members of this transcription factor family.

Using a murine model of pyelonephritis, we found that $E$. coli lacking marA, soxS and rob was unable to maintain colonization of the kidney. In this model, the bladders of diuresed mice were infected with bacteria. Subsequently, the organisms migrated to the kidneys in a process that mimics the clinical course of pyelonephritis. When the individual loci were restored in single copies, each was capable of restoring wild-type virulence.

\section{METHODS}

Bacterial strains and plasmids. The bacterial strains and plasmids used in this study are listed in Table 1. E. coli KM-D is a clinical isolate obtained from an intestinal fistula, and bears a mutated marR, resulting in a Mar phenotype (Maneewannakul \& Levy, 1996). E. coli C189 is a clinical cystitis isolate that is multidrug

Table 1. Bacterial strains and plasmids used in this study

\begin{tabular}{|c|c|c|}
\hline $\begin{array}{l}\text { Strain (serotype if relevant) } \\
\text { or plasmid }\end{array}$ & Relevant characteristics/genotype & Source or reference \\
\hline \multicolumn{3}{|l|}{ E. coli strains } \\
\hline 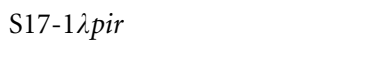 & $\begin{array}{l}\operatorname{lamB} \mathrm{F}^{-} \text {supE44 thi-1 thr-1 leuB6 lacY1 tonA21 hsdR } \\
\text { hsdM recA pro (RP4:2-Tc:: Mu:: Km::Tn7) } \lambda \text { pir }\end{array}$ & Simon et al. (1983) \\
\hline DH5 $\alpha \lambda$ pir & 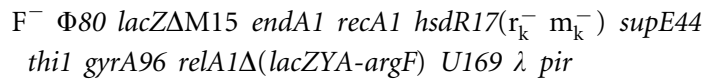 & Elliott \& Kaper (1997) \\
\hline MG1655 & $\mathrm{K}-12$ laboratory isolate & In-house strain collection \\
\hline EP-1 & Clinical isolate & Linde et al. (2000) \\
\hline KM-D & Clinical isolate, $\operatorname{mar} R$ & Intestinal fistula; Maneewannakul \& Levy (1996) \\
\hline $\mathrm{C} 189\left(\mathrm{O} 2: \mathrm{H}^{-}\right)$ & Clinical isolate & Cystitis; Yamamoto et al. (1995) \\
\hline PC1001 & KM-D $\Delta m a r A$ & This study \\
\hline PC1003 & KM-D $\Delta r o b$ & This study \\
\hline PC1005 & $\mathrm{KM}-\mathrm{D} \Delta \operatorname{soxS}$ & This study \\
\hline PC1012 & KM-D $\Delta s o x S$, rob, marA & This study \\
\hline PC1031 & PC1001:: marA & This study \\
\hline PC1033 & PC1012:: marA & This study \\
\hline PC1035 & PC1005 : : soxS & This study \\
\hline PC1037 & PC1012:: soxS & This study \\
\hline PC1038 & PC1012:: rob & This study \\
\hline PC1040 & PC1003: :rob & This study \\
\hline $\mathrm{C} 189-\mathrm{P} 2$ & C189 passaged twice in mice & This study \\
\hline PCmarA & C189-P2, marA & This study \\
\hline PCsoxS & C189-P2, soxS & This study \\
\hline PCrob & C189-P2, rob & This study \\
\hline PCrob, soxS & C189-P2, rob, soxS & This study \\
\hline PC wt Rob & C189 & This study \\
\hline PC wt MarA & C189 & This study \\
\hline \multicolumn{3}{|l|}{ Plasmids } \\
\hline pSR47s & $\mathrm{Km}^{\mathrm{R}} \mathrm{R} 6$ KoriV RP4oriT sacB & Kolter et al. (1978) \\
\hline pPC $\Delta r o b$ & pSR47s with DNA sequences flanking rob & This study \\
\hline $\mathrm{pPC} \Delta \operatorname{soxS}$ & pSR47s with DNA sequences flanking soxS & This study \\
\hline pPCAmarA & pSR47s with DNA sequences flanking marA & This study \\
\hline
\end{tabular}


susceptible (Rippere-Lampe et al., 2001; Yamamoto et al., 1995). E. coli C189-P2 was obtained following two passages of E. coli C189 in mice of the infection model described below; there was no change in drug susceptibility in the derived strain C189-P2.

Genetic techniques. In-frame (non-polar) deletions of specific genes in KM-D or C189-P2 were constructed by crossover PCR and allelic exchange (Link et al., 1997). A 1 kb DNA fragment consisting of $500 \mathrm{bp}$ flanking the upstream and downstream portions of the sequences targeted for deletion, separated by a $33 \mathrm{nt}$ spacer, was constructed by crossover PCR, and cloned into the NotI-BamHI site of the suicide vector pSR47s. pSR47s contains the R6K origin of replication, rendering it dependent on the $\pi$ protein, the kanamycinresistance gene from Tn903, and the Bacillus subtilis sacB gene, which is used as a counterselectable marker (Kolter et al., 1978). Plasmids with the cloned crossover PCR fragments were transferred

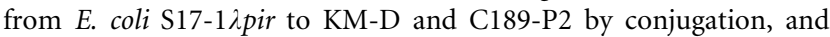
transconjugants were selected on M9 minimal medium containing $0 \cdot 2 \%$ glucose and $30 \mu \mathrm{g}$ kanamycin $\mathrm{ml}^{-1}$. KM-D and C189-P2 transconjugants were then grown overnight at $37^{\circ} \mathrm{C}$ in LuriaBertani (LB) without antibiotics. The overnight cultures were diluted in double-distilled water, and $10^{5}-10^{6}$ c.f.u. were plated on L agar containing $5 \%$ sucrose, and incubated at $30^{\circ} \mathrm{C}$ overnight. The resulting colonies were plated on LB agar with and without kanamycin. Kanamycin-sensitive colonies were tested for the presence or absence of the wild-type and deleted alleles by PCR with allele-specific primers.

The crossover PCR products used for the in-frame deletion have a 33 nt 'stuffer' sequence containing a SpeI restriction site. In order to restore the deleted genes into their original loci, the wild-type genes were amplified from KM-D and C189-P2 colonies with primers that created SpeI restriction sites at both ends of the ORF. These fragments were restricted with SpeI, and ligated to the plasmids used to make the corresponding in-frame deletions. This procedure recreates the original gene, with an additional seven amino acids (Met-Val-Ile-AsnLeu-Thr-Gly) at the amino terminus. This complementation plasmid was recombined into the chromosome of the appropriate mutant strains by allelic exchange, as described above, and the presence of the wild-type allele was confirmed by PCR.

PCR was used to identify genes specifying virulence factors known to play a role in E. coli urinary tract infection (UTI; i.e. cystitis and pyelonephritis). Primers were designed, and PCR was performed as described by others (Ruiz et al., 2002, and references therein) using total DNA isolated from C189-P2 and KM-D.

Assay for type I fimbriae expression. The assay for type I fimbriae expression was based on the protocol of Bahrani-Mougeot et al. (2002). Bacteria were grown in static LB broth for $48 \mathrm{~h}$ at $37^{\circ} \mathrm{C}$, centrifuged for $1 \mathrm{~min}$, and resuspended in $1 \mathrm{ml}$ PBS. A $25 \mu \mathrm{l}$ aliquot was then added to a flat-bottom 96-well plate. Defibrinated guinea pig blood (Colorado Serum) was centrifuged at 2300 RCF (5000 r.p.m. in an IEC Micromax microcentrifuge) for $1 \mathrm{~min}$, and washed once with PBS. The washed red blood cells were then diluted in PBS with or without $50 \mathrm{mM}$ mannose, and $25 \mu \mathrm{l}$ of this suspension was placed into the wells of a 96-well plate containing bacteria. The plate was rocked at room temperature for 15-20 min, and agglutination of the red blood cells was assessed by visual inspection of the wells, and at a magnification of $\times 100$ under an inverted microscope. E. coli EP-1 (Linde et al., 2000), which lacks the genes specifying type I fimbriae, as determined using PCR, was used as a negative control.

Ascending UTI model. All animal experiments were approved by our Institutional Animal Care and Use Committee. To make E. coli C189 suitable for studies in mice, it was passed twice through the murine host, as described below, and the resulting strain was designated C189-P2, to designate two in vivo passages. E. coli KM-D did not require these steps. The murine model of ascending pyelonephritis has been described by Hopkins et al. (1998). Briefly, CD-1 female mice (mean weight 20-30 g) were diuresed (diuresis was necessary for consistent kidney infections) on a diet consisting of water containing $5 \%$ glucose and restricted solid food (10-12 g per cage of five animals). On the day of the experiment, each mouse was anaesthetized with isoflurane, and the abdominal area was shaved and bathed with iodine, followed by sterilization with 2-propanol. A small incision was made through the outermost skin layer just above the urethra. Once the inner skin layer was exposed, another incision was made through the peritoneum, exposing the inner cavity and the bladder. A small puncture was made in the bladder to aspirate excess urine, and the infectious dose $(100 \mu \mathrm{l})$ was introduced by intravesicular inoculation.

In order to investigate the growth conditions necessary to achieve reproducible kidney infections in the UTI model, mice were infected with E. coli KM-D grown under different conditions in vitro. Bacteria grown overnight in LB broth produced the most robust and reproducible infections. From an overnight culture grown at $37^{\circ} \mathrm{C}$ in LB medium, bacteria were washed with PBS, diluted to $10^{8}$ c.f.u. $\mathrm{ml}^{-1}$ in $\mathrm{PBS}$, and $100 \mu \mathrm{l}$ of this culture $\left(10^{7}\right.$ c.f.u.) was used to inoculate the mice.

After a designated period of infection, mice were killed, and their kidneys were removed. Individual kidneys were weighed, and then suspended in $5 \mathrm{ml}$ sterile PBS. The kidneys were homogenized, and 1:10 serial dilutions were plated on MacConkey agar to determine the infectious load (c.f.u.) per gram of kidney. All data are presented as median $\log _{10}$ c.f.u. (g kidney) ${ }^{-1}$.

Statistical analyses. The statistical significance of differences between bacterial isolates was determined using ANOVA with normally distributed data, and with Dunnett's post-hoc tests. When no bacteria were recovered at a $10^{-2}$ dilution, a value of 1 was assigned to the c.f.u. (g kidney) $)^{-1}$ in order to apply statistics using parametric models. $P$ values of $<0 \cdot 05$ were considered significant.

\section{RESULTS AND DISCUSSION}

\section{Virulence of wild-type and mutant bacteria}

To investigate defects in virulence among bacterial strains lacking MarA, SoxS and/or Rob, we used a mouse model of ascending pyelonephritis. Since this model permits a determination of c.f.u. (g kidney) ${ }^{-1}$, it enabled us to make quantitative assessments of bacterial load.

Since MarA, SoxS and Rob can regulate similar genes, there is redundancy among their regulons (see above). Therefore, to mitigate potential compensatory changes, we began our experiments with a strain deleted of all three genes. Both KM-D (parent) and PC1012 (bearing in-frame deletions in marA, soxS and rob) were cultured from the kidneys of mice for up to 3 days post-infection (Fig. 1a, b). After this period, KM-D maintained colonization of the kidneys for at least 11 days post-infection, while PC1012 was cleared from the kidney after day 3 (Fig. 1b); this difference was statistically significant $(P<0 \cdot 01)$. These data suggest that the ability of PC1012 to reach the kidney (from the bladder) is not compromised, but that this strain is defective in its ability to maintain colonization of the kidney once there. 


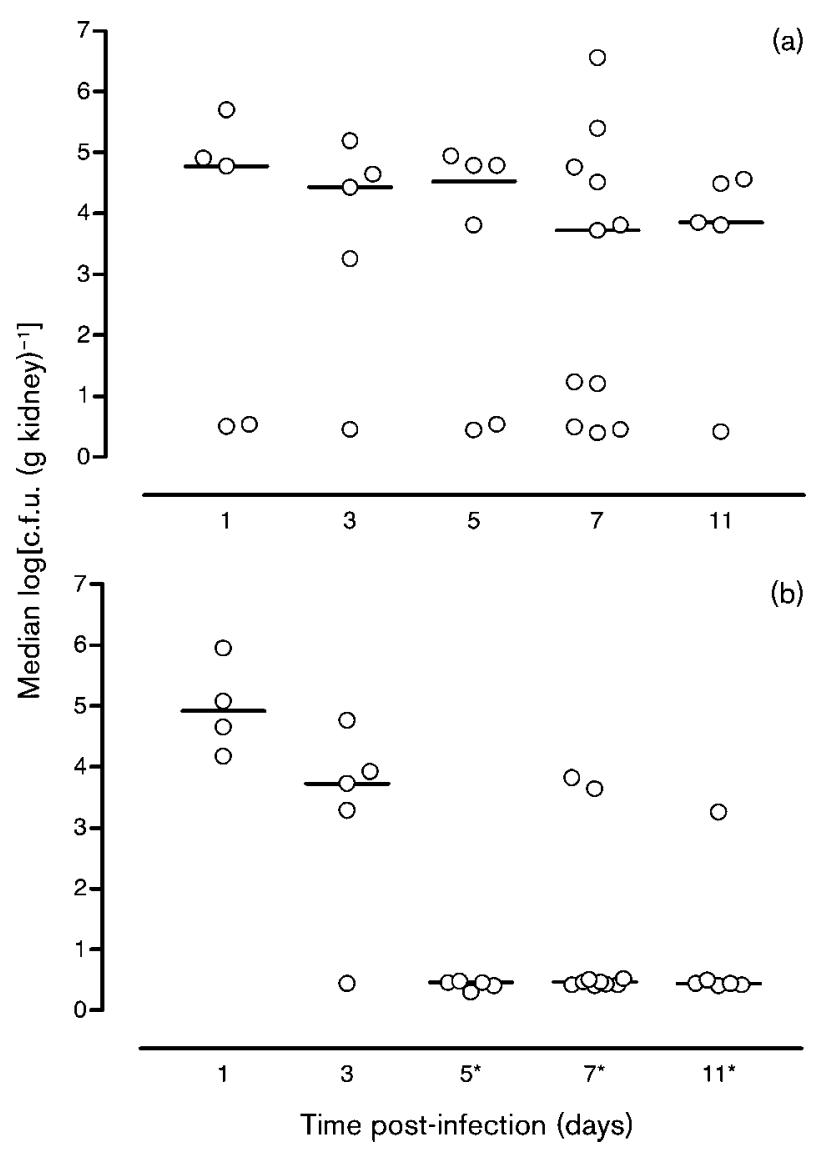

Fig. 1. Time course of kidney colonization in the mouse UTI model. Mice were infected via intravesicular inoculation (see Methods), kidneys were harvested, and bacterial loads were determined at the designated time points. (a) E. coli KM-D; no significant difference $(P>0.05)$ for day 1 versus days $3,5,7$ and 11. (b) E. coli $\mathrm{PC} 1012$ (triple deletion); ${ }^{\star} P<0.01$ for day 1 versus days 5,7 and 11 .

marA, soxS and rob were then restored individually in their original locations in the chromosome of PC1012. Complementation with single chromosomal copies of each transcription factor resulted in a strain that colonized the kidney to a similar extent as the wild-type host KM-D (compare PC1012 with PC1033, PC1037 and PC1038 in Fig. 2a). Thus, marA, soxS or rob, alone, is sufficient in restoring kidney colonization in the absence of the other two genes.

We next determined whether deletion of a single transcription factor affected kidney colonization. Deletion of soxS or rob produced an organism that exhibited a significant $(P<0 \cdot 05)$ defect in kidney colonization (compare KM-D with PC1005 and PC1003, Fig. 2b). A statistically significant effect $(P>0 \cdot 05)$, however, was not seen in the strain deleted for marA (compare KM-D with PC1001, Fig. 2b). In the case of PC1005 and PC1003, the colonization capacity of the host was fully restored when the single chromosomal copy of either soxS or rob was replaced in the respective
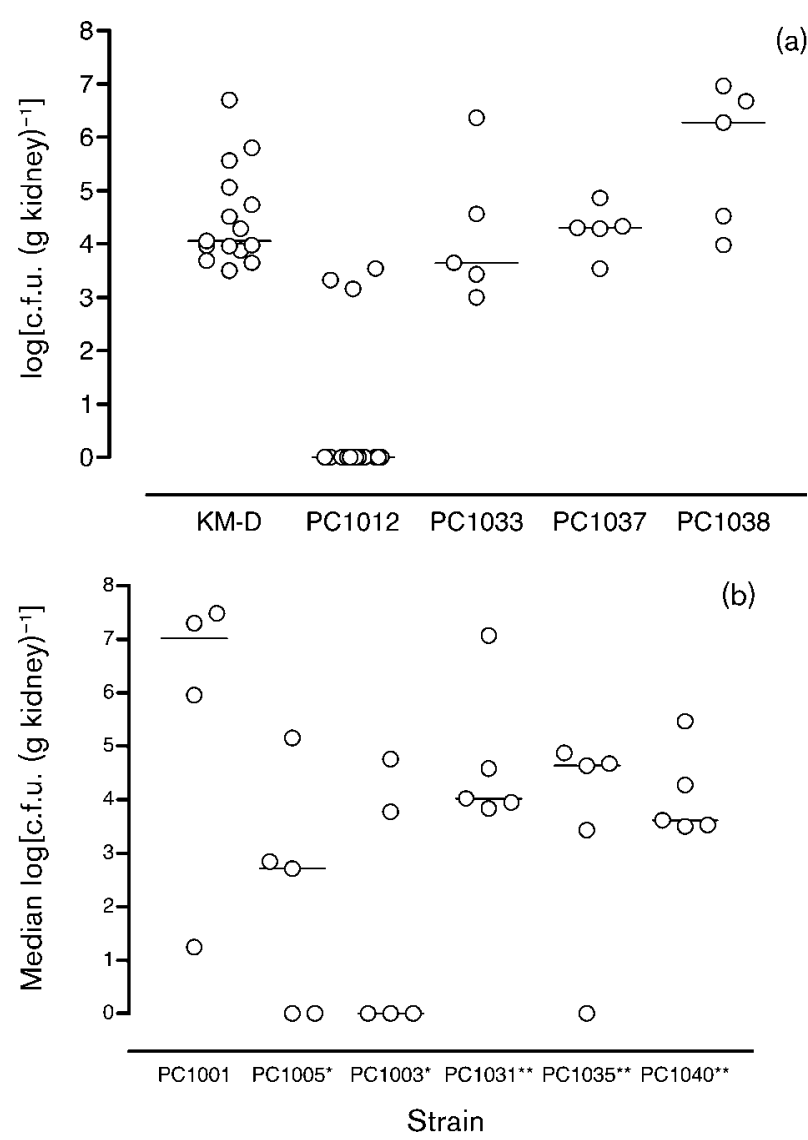

Fig. 2. Effects of transcription factor deletion on $E$. coli colonization in the KM-D genetic background in an ascending murine model of pyelonephritis. Mice were infected (see Fig. 1 legend), and kidneys were harvested at 5 days post-infection. (a) Complementation of the triple deletion (PC1012) strain; ${ }^{\star} P<0.05$ versus KM-D with $\operatorname{mar} A$, soxS or rob. The data for KM-D and PC1012 were pooled from three separate experiments. (b) Comparison of single-deletion strains [PC1001 $(\Delta \operatorname{mar} A), \mathrm{PC} 1005(\Delta s o x S)$ and $\mathrm{PC} 1003(\Delta r o b) ;{ }^{*} P \leqslant 0.05$ versus KM-D; Fig. 2a], and complementation of strains deleted for a single transcription factor [PC1031 (+marA), PC1035 $(+\operatorname{sox} S)$ and PC1040 (+rob); ${ }^{\star \star} P>0.05$ versus KM-D; Fig. 2a]. The analyses of the deletion strains and the complemented mutants were investigated in independent experiments. The relative virulence of these strains is compared with the controls (KM-D and PC1012) in (a).

single-deletion strains (compare KM-D with PC1035 and PC1040, Fig. 2b). Thus, while SoxS and Rob appear to be more critical than MarA for kidney colonization in KM-D, each restored kidney colonization in the triple mutant.

A series of single genetic deletions was constructed in E. coli C189, which is an authentic uropathogenic E. coli (UPEC) isolate obtained from a patient with cystitis (Rippere-Lampe et al., 2001; Yamamoto et al., 1995). Similar to the results observed with KM-D, deletion of the gene specifying either SoxS or Rob, but not MarA, in the C189 background, compromised the colonization capacity of the bacterium. 
The median c.f.u. (g kidney) $)^{-1}$ obtained following infection with either the single soxS or rob deletion mutant was 1 , whereas a median of $1.4 \times 10^{4}$ c.f.u. (g kidney) $)^{-1}$ was recovered following infection with the marA mutant (data not shown), which was similar to the wild-type strain. A statistically significant difference $(P<0 \cdot 05$, using a nonparametric Wilcoxon method), however, was only achieved with the soxS mutant (data not shown).

These findings strongly suggest that the colonization defect seen in the mutants is associated with the absence of SoxS or Rob, and they suggest little, if any, role for MarA if either SoxS or Rob is present. That a detrimental effect following deletion of marA alone in either E. coli strain was not seen may reflect 'backup' activity of Sox and/or Rob for critical genes regulated by the transcription factors. However, the marRAB operon is induced during growth of UPEC in another mouse model of UTI (Snyder et al., 2004).

To rule out that the genetic manipulations needed to produce the deletion strains might have a detrimental effect on the ability of the organism to colonize the murine kidneys, we investigated the colonization capacity of two wild-type strains that were obtained during the procedure used to produce the in-frame knockouts. The mutagenic process used in this study (Link et al., 1997) resulted in a population of bacteria in which half could be wild-type, and the other half mutant. Two wild-type strains, $\mathrm{PC}$ wt Rob and PC wt MarA, were indistinguishable from C189-P2 in their ability to colonize the murine kidney $\left(2 \cdot 0 \times 10^{4}\right.$ and $1.2 \times 10^{4}$, versus $1.6 \times 10^{4}$ median c.f.u. (g kidney) $)^{-1}$, respectively; data not shown).

There is a limitation of this murine UTI model. Within the experiments, some mice maintained colonization with the deletion strain PC1012 (e.g. see Fig. 1b). Similar findings of 'outliers' have also been observed with Proteus mirabilis UreR mutants (Dattelbaum et al., 2003), and when the model has been used to test antibiotics, including an aminoglycoside, a penicillin, a cephalosporin, or trimethoprim-sulfamethoxazole, to treat susceptible $E$. coli infection (Hvidberg et al., 2000; Kerrn et al., 2003; Schilling et al., 2002). Complete sterilization of any portion (urine, bladders or kidneys) of the mouse urinary tract has not been achieved in this model using genetic or therapeutic means (Dattelbaum et al., 2003; Hvidberg et al., 2000; Kerrn et al., 2003; Schilling et al., 2002).

The use of relatively large $(100 \mu \mathrm{l})$ volumes in intravesicular inoculations, i.e. directly into the bladder, can result in vesicoureteral reflux (VUR) in the mouse (Hopkins et al., 1995). While some have used intravesicular inoculation (Brzuszkiewicz et al., 2006; Hopkins et al., 1998), others have relied on the use of catheters to introduce bacteria directly into the bladder via the urethra. We tried unsuccessfully to use catheters for intraurethral infections. We do not think that VUR complicates the interpretation of our results for the following reasons. The time course of colonization was followed out to a period of 11 days, and at this point the preponderance of the murine kidneys was colonized by wild-type bacteria (Fig. 1a). Any short-term effect that might be manifested by VUR would be negated during this extended period of time. Also, since the mutants were analysed in the same model, they served as controls: they reached and colonized the kidney for up to 3 days, but were unable to maintain colonization thereafter.

The presence of bacteria within an otherwise sterile mouse kidney, nevertheless, correlates well with an active infection (Hvidberg et al., 2000). The model used in this study reports on the inability of $E$. coli deletion mutants to colonize the mouse kidney; colonization is a critical component required for most bacterial infections.

\section{Genetic, biochemical and phenotypic characterization of $E$. coli strains}

PCR was used to determine the presence of a number of genes known to be involved in the virulence of UPEC. We compared the results obtained using total DNA from C189$\mathrm{P} 2$ and KM-D. The aer gene (specifying the aerobactin siderophore), or the fimA and fimH genes (encoding the type I fimbriae), were present in both of the clinical isolates (Table 2). The UPEC isolate C189-P2 contained many of the other genetic sequences sought (Table 2). The gene afa (encoding afimbrial adhesions) was not detected by PCR in C189-P2, KM-D or E. coli K-12 MG1655. C189-P2, as reported, also lacks hly (Yamamoto et al., 1995); KM-D lacks $h l y$ and a number of genes specifying many of the known UPEC virulence factors (Table 2), but it colonized the mouse kidney to a similar extent as C189-P2. Moreover, both KM-D and C189-P2 showed a similar decrease in colonization in the mouse UTI model when either soxS or

Table 2. Virulence factors present, as determined using $\mathrm{PCR}$, in strains included in this study

Gene products are specified in parentheses. The genes afa (afimbrial adhesions) and hly (haemolysin) were not found in either C189 or KM-D. The genes fimA and fimH, but not any of the other virulence-associated genes, were also present in E. coli $\mathrm{K}-12$, a laboratory isolate. +, Present; -, not present.

\begin{tabular}{|lcc|}
\hline \multirow{2}{*}{ Gene } & \multicolumn{2}{c|}{ Host } \\
\cline { 2 - 3 } & C189 & KM-D \\
\hline aer (aerobactin) & + & + \\
cnf (cytotoxic necrotizing factor) & + & - \\
fimA (type 1 pili) & + & + \\
fimH (type 1 pili) & + & + \\
papC (pyelonephritis-associated pili) & + & - \\
papE/F (pyelonephritis-associated pili) & + & - \\
papGII (pyelonephritis-associated pili) & + & - \\
sat (autotransporter toxin) & + & - \\
sfa (S fimbriae) & + & - \\
traT (serum resistance) & + & + \\
\hline
\end{tabular}


rob was deleted. The traTgene (specifying a protein involved in serum resistance; Sukupolvi \& O'Connor, 1990), another E. coli virulence factor, was also detected by PCR in both KM-D and C189-P2 (Table 2).

Based on the results from PCR, aer, fimA and fimH, and traT seemed to be virulence candidates that were potentially affected by the deletions, since they were present in both KM-D and C189-P2. We initially ruled out aer, since we reasoned that affecting any one siderophore (if others were present) would not have such a dramatic effect on colonization in vivo (Torres et al., 2001). A role for siderophore-related functions, e.g. IroN, however, has been established using in vivo competition experiments (to detect subtle changes in virulence) between wild-type and mutant bacteria in a mouse model of UTI (Russo et al., 2002). The roles of the fimA, fimH and traT gene products were further investigated using a limited set of virulence assays (see below).

In order to assess the integrity of the bacterial outer membrane, the LPS content of KM-D, and its triple deletion mutant PC1012, was qualitatively examined using bacteria grown in LB or M9/glucose broth, or from cells isolated directly from LB agar plates (Westphal \& Jann, 1965). No differences were seen in either the LPS core or the O-antigen between the two strains on silver-stained SDS-PAGE gels (data not shown). The integrity of the cell envelope was further tested by measuring susceptibility to SDS and crystal violet in a standard disk diffusion assay using drugimpregnated paper disks. Both KM-D and PC1012 were resistant to SDS, and exhibited the same zone of inhibition $(\sim 1 \mathrm{~cm})$ when incubated in the presence of a disk containing crystal violet (data not shown).

KM-D, C189-P2, PC1012 and PCrob were tested for their susceptibility to active mouse serum, and their ability to grow in heat-inactivated mouse serum. No differences were seen (data not shown), suggesting that the expression of TraT is not involved in the colonization defect exhibited by PC1012 and PCrob.

The expression of type I pili was monitored qualitatively using mannose-sensitive agglutination of guinea pig red blood cells. Both C189-P2 and KM-D caused haemagglutination. This property was not seen with E. coli EP-1, which lacks type I fimbriae. The agglutination was abrogated by mannose, the natural substrate of type I fimbriae, indicating a specific effect of type I fimbriae (data not shown). The PCrob, soxS (double deletion) and PC1012 (triple deletion) mutants caused haemagglutination at levels similar to their wild-type parent strains (data not shown). In all instances (except for the EP-1 strain), increasing the bacterial concentration 10-fold did not result in additional haemagglutination, and reducing the bacterial concentration 10fold largely eliminated haemagglutination for all isolates (data not shown). Thus, the expression of type I fimbriae does not appear to be dependent on the presence of MarA, SoxS or Rob.
Relative to wild-type organisms, the mutant strains produced in this study did not show any statistically significant differences in growth rate, cytotoxicity toward tissue culture cells, adherence and internalization in cell culture (Garrity-Ryan et al., 2000), and induction of cytokine expression (Hedlund et al., 1999) (data not shown).

Using a limited set of known virulence assays, we were unable to find a specific defect that would account for the lack of kidney colonization, or the difference between the marA-deletion strains and strains deleted of the other two transcription factors. Given the number of genes regulated by SoxS (Pomposiello \& Demple, 2000; Pomposiello et al., 2001) and Rob, the inability to find a defect in one particular virulence factor was not entirely unexpected. Since MarA, SoxS and Rob are not required for growth of the organism in vitro, it may simply be that a regulatory defect in many genes simultaneously has as much of an effect on growth in vivo as does the removal of a single critical virulence factor, e.g. type I fimbriae. For example, it is known that some of the genes regulated by MarA are involved in biofilm formation (Otto et al., 2001), and this phenotype has been shown recently to be important for the pathogenesis of UTI in mice (Anderson et al., 2003).

The antibiotic-resistance phenotype observed in strains overexpressing MarA, SoxS and Rob depends largely on the AcrAB-TolC efflux system (Alekshun \& Levy, 1999). Although multidrug-resistance efflux systems have been shown to affect virulence (Piddock, 2006), a connection between MarA, SoxS and Rob, and efflux in the present work, is not clear. Moreover, since the wild-type and deletion strains grew equally well on MacConkey agar, a difference in susceptibility to bile salts was also ruled out, as was a major defect in AcrAB-TolC expression in vitro.

\section{Concluding remarks}

While a number of phenotypes have been assigned to MarA, SoxS and Rob transcription factors in vitro, e.g. resistance to antibiotics, household disinfectants and oxidative stress agents (Alekshun \& Levy, 1999), this is one of the first reports to demonstrate an effect of these proteins in vivo. That a family of bacterial transcription factors, which are not required for growth in vitro, has been shown to be necessary for persistence in a mouse model of UTI suggests that the factors might be exploited as new therapeutic targets. Smallmolecule inhibitors of these transcription factors (Alekshun \& Levy, 2005) can be designed to be used alone to prevent infection, or in conjunction with an antibiotic to help treat an infection. A recent study has extended this concept to $V$. cholerae infection (Hung et al., 2005).

\section{ACKNOWLEDGEMENTS}

The authors would like to thank Robert D. Arbeit for his assistance with the statistical analyses. 


\section{REFERENCES}

Alekshun, M. N. \& Levy, S. B. (1999). The mar regulon: multiple resistance to antibiotics and other toxic chemicals. Trends Microbiol $7,410-413$.

Alekshun, M. N. \& Levy, S. B. (2004). The Escherichia coli mar locus - antibiotic resistance and more. ASM News 70, 451-456.

Alekshun, M. N. \& Levy, S. B. (2005). Targeting virulence to prevent infection: to kill or not to kill? Drug Discov Today 1, 483-489.

Anderson, G. G., Palermo, J. J., Schilling, J. D., Roth, R., Heuser, J. \& Hultgren, S. J. (2003). Intracellular bacterial biofilm-like pods in urinary tract infections. Science 301, 105-107.

Ariza, R. R., Li, Z., Ringstad, N. \& Demple, B. (1995). Activation of multiple antibiotic resistance and binding of stress-inducible promoters by Escherichia coli Rob protein. J Bacteriol 177, 1655-1661.

Bahrani-Mougeot, F. K., Buckles, E. L., Lockatell, C. V., Hebel, J. R., Johnson, D. E., Tang, C. M. \& Donnenberg, M. S. (2002). Type 1 fimbriae and extracellular polysaccharides are preeminent uropathogenic Escherichia coli virulence determinants in the murine urinary tract. Mol Microbiol 45, 1079-1093.

Barbosa, T. M. \& Levy, S. B. (2000). Differential expression of over 60 chromosomal genes in Escherichia coli by constitutive expression of MarA. J Bacteriol 182, 3467-3474.

Bennik, M. H., Pomposiello, P. J., Thorne, D. F. \& Demple, B. (2000). Defining a rob regulon in Escherichia coli by using transposon mutagenesis. J Bacteriol 182, 3794-3801.

Bieber, D., Ramer, S. W., Wu, C. Y., Murray, W. J., Tobe, T., Fernandez, R. \& Schoolnik, G. K. (1998). Type IV pili, transient bacterial aggregates, and virulence of enteropathogenic Escherichia coli. Science 280, 2114-2118.

Bina, J., Zhu, J., Dziejman, M., Faruque, S., Calderwood, S. \& Mekalanos, J. (2003). ToxR regulon of Vibrio cholerae and its expression in vibrios shed by cholera patients. Proc Natl Acad Sci U S A 100, 2801-2806.

Brzuszkiewicz, E., Bruggemann, H., Liesegang, H. \& 10 other authors (2006). How to become a uropathogen: comparative genomic analysis of extraintestinal pathogenic Escherichia coli strains. Proc Natl Acad Sci U S A 103, 12879-12884.

Champion, G. A., Neely, M. N., Brennan, M. A. \& DiRita, V. J. (1997). A branch in the ToxR regulatory cascade of Vibrio cholerae revealed by characterization of toxT mutant strains. Mol Microbiol 23, 323-331.

Dattelbaum, J. D., Lockatell, C. V., Johnson, D. E. \& Mobley, H. L. (2003). UreR, the transcriptional activator of the Proteus mirabilis urease gene cluster, is required for urease activity and virulence in experimental urinary tract infections. Infect Immun 71, 1026-1030.

Elliott, S. J. \& Kaper, J. B. (1997). Role of type 1 fimbriae in EPEC infections. Microb Pathog 23, 113-118.

Eriksson, S., Lucchini, S., Thompson, A., Rhen, M. \& Hinton, J. C. (2003). Unravelling the biology of macrophage infection by gene expression profiling of intracellular Salmonella enterica. Mol Microbiol 47, 103-118.

Fetherston, J. D., Bearden, S. W. \& Perry, R. D. (1996). YbtA, an AraC-type regulator of the Yersinia pestis pesticin/yersiniabactin receptor. Mol Microbiol 22, 315-325.

Finlay, B. B. \& Falkow, S. (1997). Common themes in microbial pathogenicity revisited. Microbiol Mol Biol Rev 61, 136-169.

Flashner, Y., Mamroud, E., Tidhar, A. \& 10 other authors (2004). Generation of Yersinia pestis attenuated strains by signature-tagged mutagenesis in search of novel vaccine candidates. Infect Immun $\mathbf{7 2}$ 908-915.
Frota, C. C., Papavinasasundaram, K. G., Davis, E. O. \& Colston, M. J. (2004). The AraC family transcriptional regulator Rv1931c plays a role in the virulence of Mycobacterium tuberculosis. Infect Immun 72, 5483-5486.

Garrity-Ryan, L., Kazmierczak, B., Kowal, R., Comolli, J., Hauser, A. \& Engel, J. N. (2000). The arginine finger domain of ExoT contributes to actin cytoskeleton disruption and inhibition of internalization of Pseudomonas aeruginosa by epithelial cells and macrophages. Infect Immun 68, 7100-7113.

George, A. M. \& Levy, S. B. (1983a). Amplifiable resistance to tetracycline, chloramphenicol, and other antibiotics in Escherichia coli: involvement of a non-plasmid-determined efflux of tetracycline. J Bacteriol 155, 531-540.

George, A. M. \& Levy, S. B. (1983b). Gene in the major cotransduction gap of the Escherichia coli $\mathrm{K}-12$ linkage map required for the expression of chromosomal resistance to tetracycline and other antibiotics. J Bacteriol 155, 541-548.

Hauser, A. R., Kang, P. J. \& Engel, J. N. (1998). PepA, a secreted protein of Pseudomonas aeruginosa, is necessary for cytotoxicity and virulence. Mol Microbiol 27, 807-818.

Hava, D. \& Camilli, A. (2002). Large-scale identificationof serotype 4 Streptococcus pneumoniae virulence factors. Mol Microbiol 45, 1389-1406.

Hedlund, M., Wachtler, C., Johansson, E., Hang, L., Somerville, J. E., Darveau, R. P. \& Svanborg, C. (1999). P fimbriae-dependent, lipopolysaccharide-independent activation of epithelial cytokine responses. Mol Microbiol 33, 693-703.

Hopkins, W. J., Hall, J. A., Conway, B. P. \& Uehling, D. T. (1995). Induction of urinary tract infection by intraurethral inoculation with Escherichia coli: refining the murine model. J Infect Dis 171, 462-465.

Hopkins, W. J., Gendron-Fitzpatrick, A., Balish, E. \& Uehling, D. T. (1998). Time course and host responses to Escherichia coli urinary tract infection in genetically distinct mouse strains. Infect Immun 66, 2798-2802.

Hung, D. T., Shakhnovich, E. A., Pierson, E. \& Mekalanos, J. J. (2005). Small-molecule inhibitor of Vibrio cholerae virulence and intestinal colonization. Science 310, 670-674.

Hvidberg, H., Struve, C., Krogfelt, K. A., Christensen, N., Rasmussen, S. N. \& Frimodt-Moller, N. (2000). Development of a long-term ascending urinary tract infection mouse model for antibiotic treatment studies. Antimicrob Agents Chemother 44, 156-163.

Kerrn, M. B., Frimodt-Moller, N. \& Espersen, F. (2003). Effects of sulfamethizole and amdinocillin against Escherichia coli strains (with various susceptibilities) in an ascending urinary tract infection mouse model. Antimicrob Agents Chemother 47, 1002-1009.

Kolter, R., Inuzuka, M. \& Helinski, D. R. (1978). Trans-complementation-dependent replication of a low molecular weight origin fragment from plasmid R6K. Cell 15, 1199-1208.

Koutsolioutsou, A., Martins, E. A., White, D. G., Levy, S. B. \& Demple, B. (2001). A soxRS-constitutive mutation contributing to antibiotic resistance in a clinical isolate of Salmonella enterica (serovar Typhimurium). Antimicrob Agents Chemother 45, 38-43.

Linde, H. J., Notka, F., Metz, M., Kochanowski, B., Heisig, P. \& Lehn, N. (2000). In vivo increase in resistance to ciprofloxacin in Escherichia coli associated with deletion of the C-terminal part of marR. Antimicrob Agents Chemother 44, 1865-1868.

Link, A. J., Phillips, D. \& Church, G. M. (1997). Methods for generating precise deletions and insertions in the genome of wildtype Escherichia coli: application to open reading frame characterization. J Bacteriol 179, 6228-6237.

Maneewannakul, K. \& Levy, S. B. (1996). Identification of mar mutants among quinolone-resistant clinical isolates of Escherichia coli. Antimicrob Agents Chemother 40, 1695-1698. 
Martin, R. G. \& Rosner, J. L. (2003). Analysis of microarray data for the marA, soxS, and rob regulons of Escherichia coli. Methods Enzymol 370, 278-280.

Martin, R. G., Gillette, W. K. \& Rosner, J. L. (2000). Promoter discrimination by the related transcriptional activators MarA and SoxS: differential regulation by differential binding. Mol Microbiol 35, 623-634.

Otto, K., Norbeck, J., Larsson, T., Karlsson, K. A. \& Hermansson, M. (2001). Adhesion of type 1-fimbriated Escherichia coli to abiotic surfaces leads to altered composition of outer membrane proteins. J Bacteriol 183, 2445-2453.

Piddock, L. J. (2006). Multidrug-resistance efflux pumps - not just for resistance. Nat Rev Microbiol 4, 629-636.

Pomposiello, P. J. \& Demple, B. (2000). Identification of SoxSregulated genes in Salmonella enterica serovar Typhimurium. J Bacteriol 182, 23-29.

Pomposiello, P. J., Bennik, M. H. \& Demple, B. (2001). Genomewide transcriptional profiling of the Escherichia coli responses to superoxide stress and sodium salicylate. J Bacteriol 183, 3890-3902.

Randall, L. P. \& Woodward, M. J. (2001). Role of the mar locus in virulence of Salmonella enterica serovar Typhimurium DT104 in chickens. J Med Microbiol 50, 770-779.

Rippere-Lampe, K. E., O'Brien, A. D., Conran, R. \& Lockman, H. A. (2001). Mutation of the gene encoding cytotoxic necrotizing factor type $1[\mathrm{cnf}(1)]$ attenuates the virulence of uropathogenic Escherichia coli. Infect Immun 69, 3954-3964.

Ruiz, J., Simon, K., Horcajada, J. P. \& 8 other authors (2002). Differences in virulence factors among clinical isolates of Escherichia coli causing cystitis and pyelonephritis in women and prostatitis in men. J Clin Microbiol 40, 4445-4449.

Russo, T. A., McFadden, C. D., Carlino-MacDonald, U. B., Beanan, J. M., Barnard, T. J. \& Johnson, J. R. (2002). IroN functions as a siderophore receptor and is a urovirulence factor in an extraintestinal pathogenic isolate of Escherichia coli. Infect Immun 70, 7156-7160

Schilling, J. D., Lorenz, R. G. \& Hultgren, S. J. (2002). Effect of trimethoprim-sulfamethoxazole on recurrent bacteriuria and bacterial persistence in mice infected with uropathogenic Escherichia coli. Infect Immun 70, 7042-7049.
Schneiders, T., Barbosa, T. M., McMurry, L. M. \& Levy, S. B. (2003). The Escherichia coli transcriptional regulator MarA directly represses transcription of purA and hdeA. J Biol Chem 279, 9037-9042.

Simon, R., Priefer, U. \& Puehler, A. (1983). A broad host range mobilizing system for in vivo genetic engineering: transposon mutagenesis in Gram-negative bacteria. Biotechnology 1, 784-791.

Snyder, J. A., Haugen, B. J., Buckles, E. L., Lockatell, C. V., Johnson, D. E., Donnenberg, M. S., Welch, R. A. \& Mobley, H. L. (2004). Transcriptome of uropathogenic Escherichia coli during urinary tract infection. Infect Immun 72, 6373-6381.

Sukupolvi, S. \& O'Connor, C. D. (1990). TraT lipoprotein, a plasmid-specified mediator of interactions between Gram-negative bacteria and their environment. Microbiol Rev 54, 331-341.

Sulavik, M. C., Dazer, M. \& Miller, P. F. (1997). The Salmonella typhimurium mar locus: molecular and genetic analyses and assessment of its requirement for virulence. J Bacteriol 179, 1857-1866.

Torres, A. G., Redford, P., Welch, R. A. \& Payne, S. M. (2001). TonBdependent systems of uropathogenic Escherichia coli: aerobactin and heme transport and TonB are required for virulence in the mouse. Infect Immun 69, 6179-6185.

Valdivia, R. H. \& Falkow, S. (1996). Bacterial genetics by flow cytometry: rapid isolation of Salmonella typhimurium acid-inducible promoters by differential fluorescence induction. Mol Microbiol 22, 367-378.

van der Straaten, T., Zulianello, L., van Diepen, A., Granger, D. L., Janssen, R. \& van Dissel, J. T. (2004). Salmonella enterica serovar Typhimurium RamA, intracellular oxidative stress response, and bacterial virulence. Infect Immun 72, 996-1003.

Westphal, O. \& Jann, K. (1965). Bacterial lipopolysaccharides. Extraction with phenol-water and further applications of the procedure. Methods Carbohydr Chem 5, 83-91.

Wolfgang, M. C., Lee, V. T., Gilmore, M. E. \& Lory, S. (2003). Coordinate regulation of bacterial virulence genes by a novel adenylate cyclase-dependent signaling pathway. Dev Cell 4, 253-263.

Wu, J. \& Weiss, B. (1991). Two divergently transcribed genes, soxR and soxS, control a superoxide response regulon of Escherichia coli. J Bacteriol 173, 2864-2871.

Yamamoto, S., Tsukamoto, T., Terai, A., Kurazono, H., Takeda, Y. \& Yoshida, O. (1995). Distribution of virulence factors in Escherichia coli isolated from urine of cystitis patients. Microbiol Immunol 39, 401-404. 